\title{
El Comercio Justo: Factor Clave De Disminución De La Pobreza En La Provincia Los Ríos, Ecuador
}

\author{
René Mesías Villacrés Borja \\ Mónica Elizabeth Bonilla Manobanda \\ Sergio Enrique Fierro Barragán \\ Mario Enrique Escobar Gortaire \\ Universidad Estatal de Bolívar, Facultad de Ciencias Administrativas, \\ Gestión Empresarial e Informática, Avenida Ernesto Che Guevara, \\ Guaranda, Ecuador
}

Doi: 10.19044/esj.2018.v14n9p382 URL:http://dx.doi.org/10.19044/esj.2018.v14n9p382

\begin{abstract}
This paper focuses on the results of the research carried out to determine the incidence of Fair Trade as a factor in reducing the poverty of small cocoa producers in the province of Los Rios, Ecuador. It also aimed to ascertain if there are guaranteed aid that will grant them a more direct access to the market for their products, under fairer and more equitable conditions. A mixed research design was used in the study. The qualitative activity was focused on observation, interviews, compilation of documents, registers, materials, and exchanges developed with cocoa producers to collect the information and know their criteria and opinions on the conditions and standard of living. This, however, was carried out from a quantitative point of view. The greatest amount of information was achieved through a structured survey with the application of a multivariate option questionnaire applied to a sample of 177 farmers. The main results obtained revealed that Fair Trade is a key element in reducing poverty levels as long as it is a fundamental part of the territorial development plans of Decentralized Autonomous Governments at all levels. Also, trade unions provide real support to farmers and they also assert their claims using a more continuous use of development premiums, adjustment of minimum guaranteed prices, and pre-financing.
\end{abstract}

Keywords: Fair trade, poverty, farmers, cocoa production, equity

\section{Resumen}

Este artículo refleja los resultados de una investigación realizada para determinar la incidencia del Comercio Justo como factor de disminución de la pobreza de los pequeños productores de cacao en la provincia Los Ríos, 
Ecuador y para conocer si estos tienen garantizadas las ayudas que les permitan a sus productos un acceso más directo al mercado, en condiciones más justas y equitativas. Se utilizó un diseño de investigación mixto; la actividad cualitativa se centró en la observación, entrevistas, recopilación de documentos, registros, materiales e intercambios desarrollados con los productores de cacao para recolectar la información y conocer sus criterios y opiniones sobre las condiciones y nivel de vida. Desde el punto de vista cuantitativo, la mayor cantidad de información se obtuvo mediante una encuesta estructurada con la aplicación de un cuestionario de opción múltiple a una muestra de 177 agricultores. Los principales resultados obtenidos permitieron conocer que el Comercio Justo constituye un elemento clave que permite reducir los niveles de pobreza siempre y cuando el mismo sea parte fundamental de los planes de desarrollo territorial de los Gobiernos Autónomos Descentralizados a todos sus niveles y las organizaciones gremiales brinden un apoyo real a los agricultores, así como estos hagan valer sus reclamos, valiéndose del uso más continuado de las primas de desarrollo, el ajuste de los precios mínimos garantizados y el pre financiamiento.

Palabras clave: Comercio justo, pobreza, agricultores, producción de cacao, equidad

\section{Introduction}

Las prácticas comerciales deben dirigir sus esfuerzos para promover el cambio de manera de hacer negocios, buscando siempre la forma en que las pequeñas empresas prosperen y puedan contribuir a una economía sostenible donde no exista la explotación de los productores y trabajadores y se pongan tanto a las personas como al planeta en primer lugar. Con estos objetivos, la Conferencia de Naciones Unidas sobre Comercio y Desarrollo (UNCTAD, 1964), bajo el lema "Comercio, no ayuda", solicitaron la aprobación de unas reglas comerciales más justas que les garantizaran unos ingresos adecuados a su producción, evitaran los intermediarios innecesarios y facilitaran su acceso a los mercados internacionales.

Persiste en la actualidad ignorancia sobre el libre comercio y el comercio justo (CJ), así como confusión sobre la diferencia entre ellos (Brock \& Lopus, 2015). A tales efectos, la Organización Mundial del Comercio Justo (World Fair Trade Organization [WFTO], 2017) en su Conferencia de Arusha, Tanzania, adoptó la siguiente definición sobre comercio justo:

Es una asociación comercial, basada en el diálogo, la transparencia y el respeto, que busca una mayor equidad en el comercio internacional; la misma contribuye al desarrollo sostenible ofreciendo mejores condiciones comerciales a los productores y trabajadores marginados, especialmente en el Sur, y garantizando sus derechos. Las 
Organizaciones de Comercio Justo (con el respaldo de los consumidores) se comprometen activamente a apoyar a los productores, concientizarlos y hacer campaña para lograr cambios en las reglas y prácticas del comercio internacional convencional.

A decir de Coscione (2017) "el principal objetivo del movimiento del comercio justo es garantizar a los pequeños productores agrícolas y artesanales un acceso más directo al mercado para sus productos, en condiciones más justas y equitativas" (p.19). En Latinoamérica existe una Oficina Regional de WFTO, formada por 63 miembros provenientes de 13 países, cuya misión institucional es fortalecer las capacidades de incidencia, comercialización y monitoreo de los países miembros, promoviendo las relaciones de cooperación entre los mismos y con otros actores sociales, de modo a contribuir al mejoramiento de las condiciones de vida de pequeños productores de América Latina y atendiendo a los principios del CJ(WFTO-LA, 2017). En este importante aspecto se puede tomar como referencia la creación de redes locales de consumo con la intención de disminuir la dependencia hacia los mercados externos.

Barone y Frederico (2015), presentan como ejemplo práctico en este sentido la Ciudad del CJ de Pocos de Caldas, por ser el primer municipio brasileño que unió las tres esferas de la regulación social (gobierno, empresasy representantes de la sociedad civil organizada), persiguiendo como objetivo el establecimiento de "una mayor relación entre el campo y la ciudad, por medio de políticas municipales de estímulo al consumo y divulgación de los productos del CJ en un país productor" (p.60). Por ende, "el movimiento por un comercio justo y solidario es una de estas realidades que, a partir de la segunda postguerra, está originando una nueva manera de entender la producción y el consumo, y de reequilibrar las relaciones comerciales"(Coscione, 2015, p.12).

La utilización correcta del CJ debe propiciar el fortalecimiento del sector organizado de la sociedad, y de la misma forma brindar la posibilidad de revertir o aminorar los efectos de problemáticas acumuladas durante muchos años como la pobreza, la desigualdad y la marginación en que viven aún en nuestra región amplios sectores de la población (Becker \& Celis, 2015). Es así como en la Cumbre Mundial sobre Desarrollo Social realizada por la Organización de Naciones Unidas (ONU, 1995), en su segundo acuerdo, se plantea "nos comprometemos, como imperativo ético, social, político y económico de la humanidad, a lograr el objetivo de erradicar la pobreza en el mundo mediante una acción nacional enérgica y la cooperación internacional". (p.14).

La Coordinación Ecuatoriana de Comercio Justo (CECJ, 2017) nace en el año 2003 bajo la iniciativa de organizaciones bananeras en la Provincia de El Oro. Reactiva sus actividades en agosto del 2008, esta vez gracias al esfuerzo 
y la participación de organizaciones productoras de café, banano, plantas medicinales, cacao y quinua. Esta coordinación se encuentra legalmente reconocida por el Ministerio de Agricultura, Ganadería, Acuacultura y Pesca (MAGAP, 2010) y su objetivo fundamental es la representación de los Pequeños Productores Organizados a nivel local, nacional e internacional. Dicha coordinación también tiene carácter de incidencia política, social, medioambiental, cultural, etc.

Esta organización agrupa a nueve organizaciones de CJ a nivel nacional que representan a más de 6400 familias productoras de café, cacao, plantas medicinales y quinua, y es además miembro activo de la Coordinación Latinoamericana y del Caribe de Pequeños Productores de CJ (CLAC, 2017), se alinea a su filosofía y comparten los valores de la democracia y participación, la solidaridad, equidad, respeto, transparencia y responsabilidad social y ambiental.

En el caso específico del cacao, hay dos entidades que se encargan de realizar todo el soporte que requieren los exportadores: la Asociación Nacional de Exportadores de Cacao de Ecuador (ANECACAO Ecuador, 2017), que cuenta con toda la potencialidad y legalidad a sus efectos e incluye a todos los exportadores de cacao en grano y derivados que han decidido ser miembros activos de esa organización y el Instituto de Promoción de Exportaciones e Inversiones (PROECUADOR, 2017), de corte gubernamental dentro del Ministerio de Comercio Exterior, el cual tiene la responsabilidad de ejecutar las políticas y normas de promoción de exportaciones e inversiones del país, promoviendo la oferta de todos los productos tradicionales y no tradicionales y las posibilidades de inversión y/o introducción de los productos ecuatorianos en los diferentes mercados globales.

Existen además varias asociaciones y gremios de este sector en el Ecuador, tanto de productores como de exportadores entre los cuales pueden citarse la Asociación de Productores de Cacao Fino y de Aroma (APROCAFA); la Corporación de Organizaciones Productoras de Cacao Nacional Fino de Aroma del Ecuador (CONCACAO); la Corporación de Productores y Organizaciones Cacaoteras del Ecuador (CORPROC); la Unión Nacional de Organizaciones Campesinas Cacaoteras del Ecuador(UNOCACE); la Federación de Cacaoteros del Ecuador (FEDECADE), la Corporación de Agroindustriales de Cacao del Ecuador (CORPCACAO) y la Asociación Nacional de Cacaoteros (ASOCACAO).

La pobreza, según Borrás (2017) "se ha concebido desde dos perspectivas: la visión absoluta y la relativa"(p.15), pero todo depende del lado en que nos encontremos, y teóricamente, el máximo valor de desigualdad se alcanza cuando toda la población, menos una persona, tiene ingresos nulos (Serrano \& Benzaquén, 2017). Para comprender como se evalúa el indicador de pobreza en el Ecuador es necesario realizarlo desde la óptica del Instituto 
Nacional de Estadísticas (Instituto Nacional de Estadísticas [INEC], 2017), que utiliza recomendaciones internacionales para lo cual se compara el ingreso per cápita del hogar con la línea de pobreza y pobreza extrema. En junio de 2017 las mismas se ubicaron en 85,58 USD y 48,23 USD mensuales por persona respectivamente.

Los individuos cuyo ingreso per cápita es menor a la línea de pobreza son considerados pobres, y si es menor a la línea de pobreza extrema son considerados pobres extremos. Con este umbral, a nivel nacional la incidencia de la pobreza fue del $23,1 \%$ y la extrema pobreza del $8,4 \%$. "A nivel urbano la incidencia de la pobreza es del 14,6\% y la extrema pobreza del 3,9\%, mientras que en el área rural la pobreza es del 41,0\% y la pobreza extrema del 17,8\%". (INEC, 2017, p.4), por lo que es importante conocer que la pobreza por ingresos es aquella cuyo "ingreso per cápita familiar está por debajo de la línea de pobreza" (INEC, 2017, p.7).

Feres y Mancero (2001), plantean dos mecanismos que hacen posible determinar cuáles hogares son pobres. Una primera posibilidad es la evaluación "in situ" de los hogares para conocer si han logrado satisfacer sus necesidades básicas (NBI), para la cual es necesario realizar encuestas sobre los bienes y servicios de los cuales disponen. La segunda variante "consiste en medir los recursos del hogar, usualmente sus ingresos o sus gastos, y estimar si estos son suficientes para que el hogar pueda gozar de un nivel de vida aceptable, de acuerdo con los estándares sociales prevalecientes" (p.7).

Como limitación del método de las NBI, se puede notar que usualmente estas son evaluadas fundamentalmente en base a algunas características de la vivienda (tipo de materiales, acceso a agua potable, a sistema de eliminación de excretas o número de cuartos) y a ciertos rasgos demográficos del hogar (número de miembros, asistencia escolar de los menores, o edad, nivel educativo y condición de ocupación del jefe de hogar). De esta forma, el concepto de pobreza implícito en este método se circunscribe, en la práctica, a unas pocas necesidades específicas, dejando de lado varios de los otros elementos relevantes del bienestar (Feres \& Mancero,2001).

Al concluirse el último Censo de Población y Viviendas realizado en el Ecuador(INEC, 2010), la provincia de Los Ríos se ubicó en el tercer lugar en índices de pobreza, a pesar de ser una de las provincias que posee la tierra más fértil , contando con gran producción bananera (Diario La Hora, 2011), arrocera y de cacao. Este último desde el siglo XIX era cultivado en zonas de la cuenca alta de los ríos Daule y Babahoyo, los cuales forman el Río Guayas, y era transportado hasta el puerto de Guayaquil para su exportación, razón por la cual se le dio el nombre de "cacao arriba". Este cacao se siembra a una altitud máxima de 1200 metros sobre el nivel del mar (PROECUADOR, 2017), como se estableció en la denominación de origen, para obtener la certificación de cacao arriba en el Instituto Ecuatoriano de Propiedad Intelectual (IEPI). 
En la actualidad, el Ecuador posee una gran superioridad en este producto, pues más del $70 \%$ de la producción mundial de cacao fino de aroma se encuentra en sus tierras convirtiéndonos en el mayor productor de cacao fino o de aroma del mundo. Esto ha generado una fama importante y favorable para el país, además de que su importancia en la economía se ve reflejada en que el cacao es el quinto producto más exportado por el Ecuador dentro de las exportaciones no petroleras después del banano, pescados, rosas y demás formas de oro para uso no monetario (PROECUADOR, 2013).

De acuerdo a estudios realizados por el Ministerio Coordinador de Desarrollo Social (2017), en el año 2005, desde la perspectiva de análisis territorial:

Las provincias con mayor pobreza por NBI, es decir aquellas cuyo indicador es superior al 50\%, son todas las provincias de la Amazonía; de la Sierra las provincias de Bolívar, Cañar, Cotopaxi,Chimborazo y Loja; y de la Costa, Esmeraldas, Los Ríos y Manabí. Por el contrario para el 2015, únicamente se encuentran en este grupo las provincias de Los Ríos, Manabí y Sucumbíos (p.35).

Los agricultores y medianos productores de cacao de Babahoyo siguen alarmados por los precios que en la actualidad reciben por la venta del quintal (qq) de cacao, ya que en períodos anteriores tuvo un precio superior a los 100.00USD/qq. De hecho, a mediados del año 2016, el cacao obtuvo valores que fluctuaban entre 135.00USD/qq y 140.00USD/qq, pero en la actualidad es apenas de 75.00USD/qq. Los agricultores refieren que los costos para lograr cosechar este importante producto de la provincia y del país, son realmente altos, a tal punto que en ciertos casos la ganancia es mínima o sólo se trabaja para recuperar el capital invertido.(Diario La Hora, 2017).

El precio justo que reciben las y los cacao cultores que venden a través de los canales del Comercio Justo se compone de dos elementos fundamentales: por un lado, un precio mínimo por debajo del cual se considera que las y los productores no podrían cubrir sus necesidades ni vivir dignamente, el cual constituye una red de protección en los momentos en los que el precio internacional se desploma, como suele ocurrir continuamente. Cuando se sitúa por encima de este mínimo, el precio que paga el Comercio Justo se adapta a esta referencia pero entra en juego el segundo mecanismo: el pago de una prima de desarrollo complementaria destinada a ser invertida en el bienestar de la comunidad a través de los proyectos que los propios productores decidan democráticamente. De aquí que un precio justo sea la unión de los elementos anteriormente citados.

Además de este precio e ingresos más favorables y equitativos para hombres y mujeres, el CJ aporta otros elementos beneficiosos que tienen que ver con la retribución que reciben las y los cacao cultores por su trabajo, de acuerdo a la Agencia Española de Cooperación Internacional para el Desarrollo 
(AECID,2014). En primer lugar, se logra impulsar relaciones comerciales estables y duraderas en las que tanto productores y compradores negocian las condiciones de venta que se logran mantener durante varios años y en segundo lugar, se adelanta una parte del pago incluso antes de que comience la cosecha:

Lo cual permite a las y los cacao cultores invertir en mejoras de la producción sin tener que recurrir a los canales de financiación ordinarios, por lo general inexistentes o con condiciones abusivas que les sumergen en espirales de endeudamiento. En fin, el CJ aporta a las y los productores capacitación en cuestiones técnicas pero también para fomentar su organización en cooperativas, ganando así fuerza a la hora de negociar con otros socios comerciales las condiciones de venta de sus cosechas.(p.8)

En el Ecuador, en el año 2014, el costo estimado de establecimiento de una hectárea de Cacao Fino de Aroma fue de 2,095.00USD con respecto a la estructura de costos. El $55.36 \%$ se destina a la siembra la cual incluye la compra de plantas de cacao y la mano de obra; el $15.22 \%$ se destina a lapreparación de terreno, un $12.41 \%$ es destinado a las labores culturales en donde se incluye actividades como el control de maleza; el otro $17.01 \%$ restante es destinado a la fertilización y control fitosanitario y la cosecha se da a partir del tercer año, por lo que esta actividad representa el $60 \%$ del costo total de producción (MAGAP, 2014).

De acuerdo a estudios del Banco Central de Ecuador (BCE,2016), en la provincia de Los Ríos, en los cantones: Vinces, Babahoyo, Montalvo, Urdaneta, Pueblo Viejo, Ventanas, Quinsaloma, Mocache, Buena Fe, Quevedo y San Carlos, los rendimientos que los agricultores habrían obtenido por zona productiva fueron los siguientes: Vinces $35 \mathrm{qq} / \mathrm{ha}$; Babahoyo 18qq/ha; Montalvo 25 qq/ha; Urdaneta y Pueblo Viejo 44 qq/ha; Ventanas y Quinsaloma 40 qq/ha; Quevedo, Mocache, Buena Fe y San Carlos 36 qq/ha, mientras que el costo de producción real para el cultivo de una hectárea de cacao a nivel de las zonas investigadas incrementó, como resultado del alto precio de los insumos y pesticidas, pasando de 3,500.00 USD a 3,700.00 USD.

Según los resultados agregados, el $50 \%$ de los entrevistados en ese estudio afirmaron que los agricultores aumentaron sus inversiones en el período investigado debido al incremento en el pago de la mano de obra (jornal) y el alto precio de los insumos. El precio del quintal de cacao pagado al productor, en las zonas investigadas durante 2015, registró valores promedio de 104.00 USD, con un incremento del $4 \%$ respecto al año anterior. Por su parte, el jornal promedio pagado a los trabajadores en las diversas zonas de cultivo de cacao fue de 14.00 USD con alimentación, y 16.00 USD sin alimentación (BCE, 2016). 
De acuerdo a las cifras de comercio exterior del BCE en 2015, las exportaciones en volumen de cacao experimentaron también un crecimiento de $18.7 \%$, en relación con las exportaciones del año anterior, pasando de 577,184.64 TM en 2014 a 692,849.22 TM en 2015.

En cuanto a las exportaciones en valor, se incrementaron en $20 \%$, lo que refleja el buen precio del cacao en el mercado internacional, al pasar de 577.1 millones USD en 2014 a 692.8 millones USD en 2015 (BCE, 2016).

El Secretario de la ONU, Ban Ki Moon, al referirse al cumplimento de los Objetivos de Desarrollo del Milenio, expresó "estoy profundamente consciente de que las desigualdades persisten y que el progreso ha sido desigual. La pobreza continúa concentrada predominantemente en algunas partes del mundo" (ONU, 2015, p.3), por lo que esta organización adoptó un nuevo compromiso con la humanidad, llamado Agenda 2030 para el Desarrollo Sostenible (ONU,2015b), en la cual Ecuador se comprometió participar en el cumplimiento de estas metas mediante una resolución de la Asamblea Nacional aprobada con 126 votos a favor que fomentará la promulgación de leyes, la aprobación de presupuestos y la representación de los intereses de la voluntad del pueblo soberano, con el objetivo de viabilizar el óptimo cumplimiento de esta agenda de modo articulado con el Plan Nacional de Desarrollo (Diario Andes, 2017).

\section{Metodología}

A partir de estas premisas, considerando la importancia que tiene la producción de cacao como generador de riquezas para las familias ecuatorianas, se puede afirmar que los estudios locales que se aproximan al CJ de este rubro económico y sus influencias en la disminución de la pobreza aún son insuficientes, situación que devela insuficiente conocimiento de las ventajas que brinda esta alternativa a las asociaciones y cooperativas de productores de cacao. Ante esta situación, los autores se plantearon las siguientes interrogantes para responder al problema existente en la presente investigación:

- ¿Existen y funcionan en realidad todas las organizaciones e instituciones relacionadas con la producción y comercialización del cacao con las bondades y apoyos que dicen aportar?

- ¿Están presentes las verdaderas condiciones de comercio justo para los productores cacaoteros de la provincia Los Ríos?

- ¿Cuál es el comportamiento de las necesidades básicas insatisfechas (NBI) en los productores de cacao?

- ¿Qué niveles de pobreza desde la óptica de ingresos se manifiestan en los productores de cacao de la provincia Los Ríos? 
- ¿De qué manera el CJ puede constituirse como un factor clave en la reducción de la pobreza de los pequeños productores de cacao de la provincia de Los Ríos?

Los autores consideraron los estudios realizados por Fassinger y Morrow (2013), los cuales les permitieron entender su propio estatus y posición como persona culturalmente ubicada en relación con la comunidad, especialmente en lo que se refiere al poder y el privilegio, es decir, en relación con el grupo social que será analizado, debiendo estar preparado para honrar las fortalezas y apoyar las necesidades de esas personas a medida que se manifestaban durante la realización del trabajo.

Se decidió el uso de métodos mixtos de investigación (Creswell, 2014; Morse \& Niehaus,2016; Cienfuegos, 2016; Berthod et al , 2017; Coolican, 2017; Echevarría, 2017) por representar un conjunto de procesos sistemáticos, empíricos y críticos que implican la recolección y el análisis de datos cuantitativos y cualitativos, debiendo realizarse una integración y discusión conjunta que hizo posible realizar inferencias producto de toda la información recabada; de aquí que tanto los datos cualitativos como los cuantitativos son simultáneos en su recolección, y que su análisis y su interpretación permiten lograr un mayor entendimiento del fenómeno bajo estudio (Zohrabi,2013).

Según Onwuegbuzie y Corrigan (2014) en la investigación mixta de igual estatus, el investigador adopta (aproximadamente) el mismo uso de las metodologías cuantitativa y cualitativa, aplicando diferentes instrumentos que posibiliten recolectar los datos necesarios para su evaluación. En este caso los instrumentos cualitativos fueron: observación, entrevistas, recopilación de documentos, registros, materiales e intercambios desarrollados con los productores de cacao para recolectar la información y conocer sus criterios y opiniones sobre las condiciones y nivel de vida. Los instrumentos cuantitativos fueron: cuestionarios, análisis de contenido.

Como característica distintiva de este estudio se señala la escasa existencia de literatura que aborde el problema a nivel local, lo que llevó a los autores a encontrarse en medio de una situación enigmática y de conocimiento donde se dieron perspectivas de conflicto al enfrentar la sabiduría cultural de las personas que estaban siendo estudiadas, ya que estos individuos presentaban diferentes enfoques sobre los fenómenos bajo estudio. Por esta razón, hubo que plantearse un desafío que permitiera encontrar formas de enmarcar el estudio científico de una manera que legitimara el conocimiento cultural.

En consecuencia, la mayor cantidad de información se logró obtener mediante una encuesta estructurada con la aplicación de un cuestionario de opción múltiple (López-Roldán \& Fachelli, 2015; Moser \& Kalton, 2016), elaborada por expertos (Hernández, Fernández \& Baptista, 2014) y dirigida 
fundamentalmente a los agricultores, es decir, una etapa de metodología cuantitativa (Hodge \& Gillespie, 2008) y una etapa simultánea cualitativa (Martínez, 2006; Rubin \& Babbie, 2017 ) sustentada en la realización de entrevistas a estos mismos actores. A estas etapas se une el apoyo que brindó el método análisis-síntesis en la revisión de la documentación existente para llevar a cabo el diseño de la investigación, la determinación de los presupuestos teóricos y arribar a las conclusiones planteadas.

La población de estudio estuvo constituida por los agricultores de las 22 asociaciones cacaoteras de la Provincia de Los Ríos. Mediante el muestreo probabilístico por conglomerados se seleccionó la asociación La Esmeralda del Cantón de Montalvo que cuenta con 16 sub-asociaciones y 323 agricultores de cacao. Para el cálculo de la muestra se determinó un índice de confianza del 95\% ( $\mathrm{k}=1.96)$; el error muestral deseado fue del 5\%. La proporción de individuos en la población que poseen la característica de estudio (p) se consideró igual a la de aquellos que no la poseen (q), aplicándose entonces que $\mathrm{p}=\mathrm{q}=0.5$ es la opción más segura, obteniéndose como resultado que la muestra (n) fuera de 177 personas a encuestar.

Los índices de pobreza fueron medidos mediante los ingresos del hogar y las NBI. No se considera pobre una familia que no satisfaga alguna de estas necesidades; para ello se hizo una valoración integral de todos los indicadores, los cuales se verificaron mediante la encuesta y observación directa, para determinar si los hogares habían satisfecho la mayoría de ellas.

En las NBI se tomaron en cuenta elementos como: hacinamiento, vivienda inadecuada, abastecimiento de agua, servicios sanitarios para el desecho de excretas y capacidad económica, un indicador indirecto que, aunque no mide una necesidad básica propiamente, intenta reflejar la posibilidad que tiene el hogar de obtener ingresos suficientes y su capacidad de consumo. Es necesario destacar que el acceso a una vivienda adecuada se vincula con la necesidad de las personas de protegerse del medio ambiente, así como con aspectos de privacidad e higiene, cuya ausencia deteriora considerablemente la calidad de vida. La condición de hacinamiento se mide a partir del número de personas por habitación, mientras que la calidad de la vivienda se determina en función de los materiales de construcción utilizados y condiciones de: piso, paredes y techo (INEC, 2017). Para la recolección de datos, se consideraron algunos elementos que se incluyen en la Encuesta de condiciones de vida (ECV) que aplica el INEC (2015), adecuándolos al objetivo de estudio de la presente investigación como parte del cuestionario de la encuesta que se aplicó independientemente a cada uno de los agricultores seleccionados dentro de la muestra.

Al realizar las encuestas, uno de los objetivos fue conocer hasta qué punto los agricultores poseían una vivienda digna de acuerdo a los parámetros que se evalúan para medir la pobreza. De los 177 que conforman la muestra, 
solo el $17 \%$ vive en una casa de cemento, mientras que un $64 \%$ lo hacen en casas de madera o mixtas, encontrándose un $16 \%$ que habitan en casas de caña, mientras que penosamente un 3\% tienen que hacerlo en covachas.

Si este primer análisis resulto llamativo, el siguiente paso constituyó otra alarma al revisar el estado de conservación de las viviendas, donde se evaluaron los componentes estructurales: techo, paredes y piso (Ver Fig.1). En sentido general, el estado de conservación de todas las viviendas es catalogado por los encuestados como deficiente, corroborado en el trabajo de campo mediante la observación. Un elemento que llamó poderosamente la atención es el relativo a los techos de las viviendas, pues sólo el $16.48 \%$ se cataloga como bueno, aspecto muy preocupante en una zona donde los inviernos se comportan de forma agresiva con torrenciales aguaceros. La misma calificación crítica se le otorga a paredes y pisos, donde el resultado entre regular y mal es catastrófico.

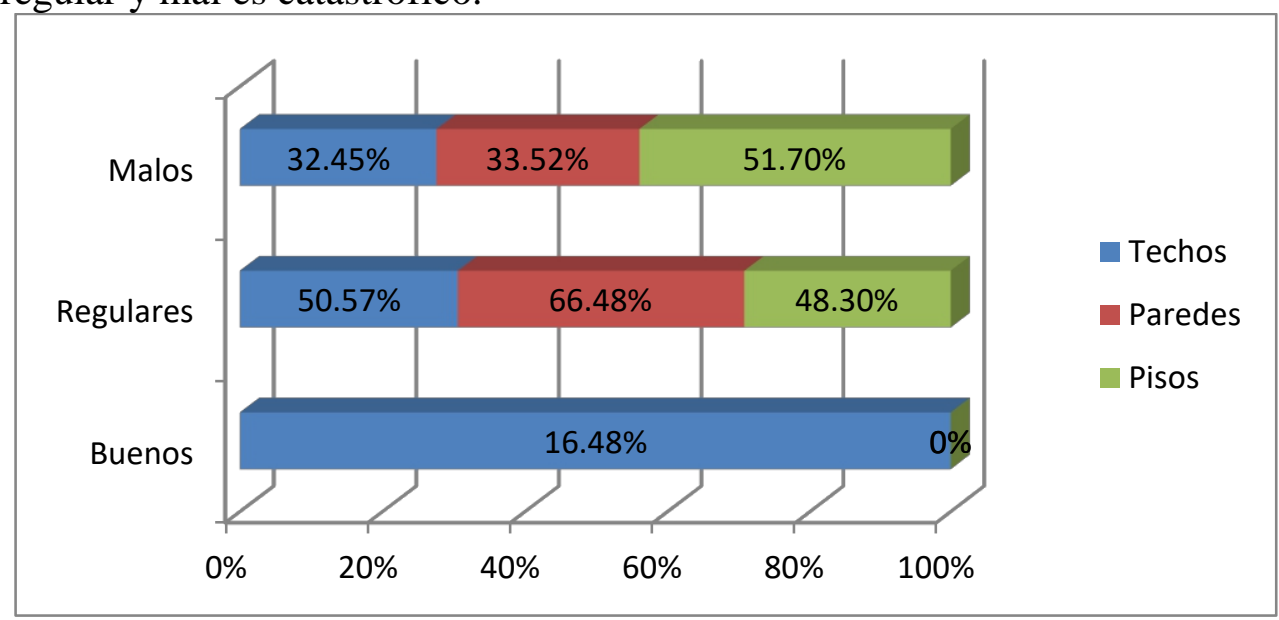

Figura 1. Estado de conservación de techos, paredes y pisos de las viviendas de los agricultores

Fuente: encuestas realizadas

Al realizar la pregunta ¿considera usted que vive en estado de hacinamiento? fue necesario explicar bien a cada persona lo que significa este elemento dentro de las valoraciones que realiza el INEC, donde se considera que existe pobreza si la relación de personas por dormitorios es mayor a tres. En este caso, el $53.1 \%$ de los encuestados respondió de forma afirmativa; es decir existe hacinamiento en la mayoría de las familias de los productores de cacao de la estructura organizativa analizada. Un $46.9 \%$ dijo no presentar esta situación e incluso, dentro de estos, un $10.1 \%$ expresó contar con tres habitaciones, lo que le permite vivir de forma holgada y fuera de los estándares de pobreza.

Respecto a la forma en que obtienen el agua para el hogar, el $48.0 \%$ lo realiza mediante pozo o grieta, un $28.5 \%$ lo obtiene mediante el sistema de 
acueducto , mientras que el $20.1 \%$ la toma directamente desde la vertiente de un río y sólo un 3.4\% la recibe mediante carros repartidores, lo que permite deducir que sólo el 31.4\% recibe un producto apto para el consumo humano. $\mathrm{Si}$ a esto se le agrega la forma en que se realiza la eliminación de las excretas se puede pensar que la situación de riesgo bilógico es latente, pues el $81.6 \%$ de los encuestados la realiza mediante pozo séptico y solo el $18.4 \%$ mediante la red pública de alcantarillado.

A la interrogante de cuál ha sido el promedio de ingresos en los últimos 24 meses, el $71.5 \%$ de los encuestados manifestó haber ganado al menos 3000.00 USD, un $15.1 \%$ expresó que habían percibido entre 3001.00 USD y 7000.00 USD, mientras el 13.4\% manifiesta un ingreso superior a los 7001.00 USD e inferior a 10000.00 USD. Este tipo de pregunta siempre despertó recelo entre los agricultores pues, a pesar de la identificación plena de los autores desde el inicio del trabajo de campo, los encuestados manifiestan que muchas veces han sido engañados y muchas informaciones anteriores se les entregan al Servicio de Rentas Internas (SRI), de aquí que el por ciento de error asumido en el cálculo de la muestra haya sido del $5 \%$.

Si se considera lo planteado por el BCE (2016) de que el costo de producción real para el cultivo de una sola hectárea de cacao debido al alto precio de los insumos y pesticidas se encuentra en la actualidad en 3700.00 USD y el $71.5 \%$ de los encuestados (126 agricultores) plantea que han tenido ingresos de al menos 3000.00 USD en los últimos 24 meses, entonces algo no debe estar bien en los datos aportados, pues a pesar de que las cifras analizadas arrojan una pérdida muy por encima de lo normal, se mantienen aún apegados a su tierra viviendo en condiciones infrahumanas, de aquí la sospecha del autor de que las cantidades expresadas en los ingresos no responden a la realidad, lo cual puede crear un sesgo en el resultado de la investigación.

Otro elemento a considerar fue el tiempo que llevan estos productores asociados al Comercio Justo. 126 agricultores $(71,5 \%)$ con ingresos inferiores a 3000.00 USD llevan menos de tres años vinculados a este tipo de comercio, de ellos $26(14.77 \%)$ llevan menos de un año, por lo que sus inversiones se encuentran en franco período de recuperación. En este sentido es significativo señalar que de los 47 productores que superan los cuatro años de afiliación, 24 (51.06\%) tienen ingresos entre 7001.00 USD y 10000.00 USD y los otros 23 restantes entre 3001.00 USD y 7000.00 USD.

De acuerdo a la información brindada por los productores de cacao el estado de sus ingresos bajo las condiciones del Comercio Justo se revela de la siguiente manera: el $6.25 \%$ considera que todo sigue igual, es decir, no experimentan ningún tipo de mejora, mientras que el $68.75 \%$ valora la asociación al CJ como algo que le ha permitido mejorar sus ingresos de forma positiva. Por otra parte, un $25 \%$ se mantiene aún a la expectativa pues consideran que aunque ha existido un ligero avance, la mejoría no está acorde 
con sus esfuerzos y expectativas. En sentido general, se califica como positiva la variante del CJ.

Los agricultores, al indagarse si percibían mejorías en relación con su asociación al CJ, y en qué aspecto lo utilizaban, lo valoraron de acuerdo como se muestra en la siguiente tabla.

Tabla 1. Mejorías en las condiciones de vida con respecto a los ingresos por asociación al Comercio Justo

\begin{tabular}{lcccccc}
\hline CATEGORÍA & SI & \% & ALGO & \% & NO & \% \\
\hline Salud & 66 & 37,29 & 53 & 29,94 & 58 & 32,77 \\
Educación & 66 & 37,29 & 53 & 29,94 & 58 & 32,77 \\
$\quad$ Servicio de agua y electricidad & 15 & 8,47 & 103 & 58,19 & 59 & 33,33 \\
Técnicas de cultivo & 18 & 10,17 & 100 & 56,50 & 59 & 33,33 \\
Asesoría de cultivos & 91 & 51,41 & 34 & 19,21 & 52 & 29,38 \\
Capacitación & 128 & 72,32 & 0 & 0 & 49 & 27,68 \\
$\quad$ Diversificación de la producción & 128 & 72,32 & 0 & 0 & 49 & 27,68 \\
\hline
\end{tabular}

Fuente: encuestas realizadas

La mejoría de vida es percibida por los agricultores con respecto a su asociación al CJen tres elementos fundamentales: la capacitación, la posibilidad de diversificar la producción y la asesoría de cultivos, donde son apoyados fundamentalmente ,de acuerdo al criterio de más del $70 \%$ de los asociados , por la Corporación de Productores y Organizaciones Cacaoteras del Ecuador (CORPROC), la Asociación de Productores de Cacao (ANECACAO), el MAGAP y el Gobierno Provincial Autónomo de Los Ríos, así como por la asesoría de la Universidad Técnica de Babahoyo(UTB). De la misma forma, consideran que existe insuficiencia aún en el apoyo a las técnicas de cultivo y el acceso a los servicios de agua y electricidad, considerando la mejoría en educación y salud como media.

$\mathrm{Al}$ indagar sobre el nivel de conocimiento que poseen los agricultores sobre dos elementos fundamentales del CJ: el precio mínimo garantizado y la prima de desarrollo, se pudo conocer que el $75.70 \%$ tienen bajo conocimiento sobre el precio mínimo garantizado, un $13,55 \%$ consideran que lo conocen medianamente y solo el 11,86\% manifiesta un nivel alto de conocimiento al respecto. Sobre el conocimiento de la prima de desarrollo el $77.40 \%$ casi no conoce nada al respecto, mientras que $11,86 \%$ expresa poseer un nivel medio de conocimiento sobre este aspecto. De igual manera se manifiestan los que consideran conocerlo bien; cuando se les interrogó acerca de cuál era la definición correcta de la prima de desarrollo el 68,92\% de los encuestados respondió incorrectamente y sólo el 31,08\% de forma correcta.

De los 177 productores encuestados, $122(68,92 \%)$ declaran que reciben primas de desarrollo a veces y $14(7.90 \%)$ siempre, los restantes 41 
$(23,16 \%)$ dijeron no recibirlas nunca. Similar situación se presenta con el pre financiamiento. Al preguntar si reciben precio mínimo garantizado el 6,77\% de los productores declaran no recibirlo, el $66,66 \%$ a veces y sólo el 26,55\% respondieron que lo recibe siempre. El 100\% de los encuestados manifestó que los ingresos por la venta de cacao son destinados en primer lugar a la compra de alimentos, el $76.50 \%$ dijo que en segundo lugar lo dedica a la educación de los hijos y en tercer lugar el $23,5 \%$ expresa que lo emplea en compra de calzado y vestimenta de las familias.

\section{Conclusiones}

- Mediante el estudio se logró comprobar que no todas las organizaciones que agrupan a los agricultores de cacao funcionan de acuerdo a los intereses y principios para lo que fueron creadas.

- Es latente la existencia del Comercio Justo en función de los agricultores, pero no se les brinda a los mismos todas las potencialidades que se necesitan para un despegue total de sus producciones que les permita lograr mejor condiciones de vida.

- No existe un conocimiento adecuado sobre las características y elementos del Comercio Justo por parte de todos los agricultores, lo que indica la necesidad de implementar acciones dirigidas a la capacitación que propicie el aprendizaje de sus particularidades.

- Desde el punto de vista macro existe una notable mejoría de la pobreza; de acuerdo a la Encuesta de Condiciones de Vida Sexta Ronda (INEC, 2014), la misma se redujo en la provincia Los Ríos al 33\%, de un $49.1 \%$ que existía en 2006 , lo que se puede constatar también en las respuestas de los agricultores donde se manifiesta que sí han logrado tener mejorías de vida al realizar su afiliación al Comercio Justo. No obstante persisten aún problemas serios con las viviendas.

- El comportamiento de las NBI es otro elemento a considerar, pues existe hacinamiento en la mayoría de los agricultores encuestados; la fuente de agua más utilizada son los pozos, y con respecto a la eliminación de las excretas sólo el $18.4 \%$ lo hace mediante la red pública de alcantarillado.

- El CJ constituye un elemento clave que permite reducir la pobreza siempre y cuando el mismo constituya parte fundamental de los planes de desarrollo territorial de los Gobiernos Autónomos Descentralizados (GAD) a todos sus niveles y las organizaciones gremiales brinden un apoyo real a los agricultores, así como estos hagan valer sus reclamos

- El estudio ha posibilitado conocer que las primas de desarrollo y el ajuste de precios mínimos garantizados, han permitido progresivas 
mejorías en los ingresos de los productores de cacao de la provincia de Los Ríos.

- Los prefinanciamiento otorgados, aunque no en las necesidades reales, han permitido un incremento de los agricultores en sus necesidades básicas, ya que en sentido general estos agricultores han partido de una situación de pobreza, incluso de pobreza extrema, y la situación hoy día es diferente, pudiendo mejorarse más.

- Existe la necesidad de implementar estrategias de capacitación que permitan a la colectividad asegurar una toma de decisión democrática en cuanto a la utilización del excedente generado y poder continuar elevando los estándares de vida de las familias.

\section{References:}

1. Agencia Española de Cooperación Internacional para el Desarrollo (2014). Coordinadora Estatal de Comercio Justo .Monográfico sobre el cacao .Cuaderno de comercio justo, 4(), Recuperado de http:/comerciojusto.org/wp-content/uploads/2014/04/cast.pdf

2. Asociación Nacional de Exportadores de Cacao- Ecuador. (2017). Recuperado de http://www.anecacao.com/index.php/es/inicio.html

3. Banco Central del Ecuador (2016). Reporte de coyuntura sector agropecuario, 88(4) Recuperado de

https:/contenido.bce.fin.ec/documentos/PublicacionesNotas/Catalog o/Encuestas/Coyuntura/Integradas/etc201504.pdf

4. Barone, M., \& Frederico, S. (2015). La ciudad brasileña del comercio justo: una experiencia pionera en POÇOS DE CALDAS. Eutopía,(7), 59-73.

5. Becker, C., \& Celis, S. (2015). Comercio justo, producción cafetalera y sociedad civil en Centroamérica. Eutopía: Revista de Desarrollo Económico Territorial, (7), 27-37.

6. Berthod, O., Grothe-Hammer, M., \& Sydow, J. (2017). Network Ethnography: A Mixed-Method Approach for the Study of Practices in Interorganizational Settings. Organizational Research Methods, 20(2), 299-323. doi: 10.1177/1094428116633872

7. Borrás, V. (2017). Multidimensionalidad de la pobreza en Uruguay, 2006-2013: análisis en cuatro dominios geográficos. Revista de Ciencias Sociales, (40), 13-42.

8. Brock, J. R., \& Lopus, J. S. (2015). A Note on Teaching About Fair Trade. The American Economist, 60(1), 74-78. https://doi.org/10.1177/056943451506000107

9. Cienfuegos, M. (2016). Lo cuantitativo y cualitativo en la investigación. Un apoyo a su enseñanza. Revista Iberoamericana para 
la Investigación y el Desarrollo Educativo. 7(13), 0-0, Recuperado de http://www.ride.org.mx/index.php/RIDE/article/view/231/1059

10. Creswell, J. (2014). Research Design: Qualitative, Quantitative, and Mixed Methods Approaches. Los Angeles, EE.UU: SAGE Publications Inc.

11. Coolican, H. (2017). Research Methods and Statistics in Psychology. Sixth edition. London, United Kingdom: Psychology Press. Taylor \& Francis Ltd

12. Conferencia de Naciones Unidas sobre Comercio y Desarrollo (1964). Recuperado de http://unctad.org/es/Docs/td97vol1_sp.pdf

13. Coordinadora Ecuatoriana de Comercio Justo. (2017). ¿Quiénes somos? Recuperado de

http://www.cecjecuador.org.ec/coordinadora/quienes-somos/

14. Coordinadora Latinoamericana y del Caribe de Pequeños Productores y Trabajadores de Comercio Justo. (2017). Quiénes somos - CLAC. Recuperado de http://clac-comerciojusto.org/que-es-la-clac/quienessomos/

15. Coscione, M. \& Mulder, N. (Ed.)(2017). El aporte del comercio justo al desarrollo sostenible. CEPAL y CLAC. Recuperado de http://repositorio.cepal.org/handle/11362/42404

16. Coscione, M. (2015). América Latina y el sentido originario del comercio justo. Eutopía: Revista de Desarrollo Económico Territorial, (7), 11-26.

17. Diario La Hora. (11 de Octubre de 2011). Los Ríos la tercera provincia más pobre del país según Censo 2010. Recuperado de https://lahora.com.ec/noticia/1101218596/los-rc3ados-la-terceraprovincia-mc3a1s-pobre-del-pac3ads-segc3ban-censo-2010

18. Diario La Hora. (10 de Julio de 2017). Preocupación por el precio del cacao. Recuperado de

https://lahora.com.ec/losrios/noticia/1102083023/preocupacion-porel-precio-del-cacao

19. Diario Andes (21 de Julio de 207). Ecuador se compromete a implementar la Agenda 2030 de Desarrollo Sostenible de la ONU. Recuperado de https://andes.info.ec/es/noticias/politica/3/ecuadorcompromete-implementar-agenda-2030-desarrollo-sostenible-onu

20. Echevarría, H. (2017). Clasificación de los diseños mixtos en las Ciencias Sociales y aplicación al análisis de tres informes de investigación. Revista Latinoamericana de Metodología de la Investigación Social.12 (6) ,8-26, Recuperado de http://www.relmis.com.ar/ojs/index.php/relmis/article/view/156

21. Fassinger,R. \& Morrow, S. (2013). Toward best practices in Quantitative, Qualitative, and Mixed-Method Research: A Social 
Justice Perspective. Journal for Social Action in Counseling and Psychology. 5(2),69-83. Recuperdo de http://psysr.org/jsacp/Fassinger_V5N2-13_69-83pdf

22. Feres,J. y Mancero, X.(2001) El método de las necesidades básicas insatisfechas (NBI) y sus aplicaciones en América Latina. Estudios Estadísticos y Prospectivos. Recuperado de http://repositorio.cepal.org/bitstream/handle/11362/4784/1/S0102117 _es.pdf

23. Hernández, R.; Fernández, C. Y Baptista, P. (2014) Metodología de la Investigación ( $6^{\text {a }}$ Edic). México D.F., México: McGrall Hill.

24. Hodge,D. \& Gillespie,D. (2008). Phrase completion scale. Journal of Social Service 10.1300/J079v33n04 01

Research.33(4),1-12. doi:

25. Instituto de Promoción de Exportaciones e Inversiones. (2013). Estudios de Mercado.

Recuperado de http://www.proecuador.gob.ec/exportadores/publicaciones/perfilesproducto-mercado/

26. Instituto Nacional de Estadística (2010). Base de Datos-Censo de Población y Vivienda 2010. Recuperado de http://www.ecuadorencifras.gob.ec/base-de-datos-censo-depoblacion-y-vivienda-2010/

27. Instituto Nacional de Estadísticas (2014) . Encuesta de condiciones de vida, sexta ronda, período 2013-2014. Recuperado de http://ecuadorencifras.gob.ec/condiciones-de-vida/

28. Instituto Nacional de Estadística (2015). Pobreza por Consumo. Recuperado de http://www.ecuadorencifras.gob.ec/pobreza-por-consumo/

29. Instituto Nacional de Estadísticas (2017) .Reporte de pobreza y desigualdad .Recuperado de http://www.ecuadorencifras.gob.ec/documentos/webinec/POBREZA/ 2017/Junio/Informe\%20pobreza\%20y\%20desigualdad\%20\%20jun\%202017\%2014072017.pdf )

30. Instituto Nacional de Estadísticas (2017). La pobreza por Necesidades Básicas Insatisfechas. Recuperado de http://www.ecuadorencifras.gob.ec/pobreza-por-necesidades-basicasinsatisfechas/

31. Instituto de Promoción de Exportaciones e Inversiones. (2017). Quienes Somos. Recuperado de http://www.proecuador.gob.ec/institucional/quienes-somos/

32. López-Roldán, P.; Fachelli, S. (2015). Metodología de la Investigación Social Cuantitativa. Bellaterra (Cerdanyola del Vallès): Dipòsit Digital 
de Documents, Universitat Autònoma de Barcelona. Recuperado de http://ddd.uab.cat/record/129382

33. Martínez, M. (2006). Validez y confiabilidad en la metodología cualitativa. Paradigma. 27(2), 7-33, Recuperado de http://revistas.upel.edu.ve/index.php/paradigma/article/view/3759/18 66

34. Ministerio Coordinador de Desarrollo Social (2017).Informe de desarrollo social 2007-2017. Recuperado de http://www.competencias.gob.ec/wpcontent/uploads/2017/06/06IGC2 017-INFORME.pdf

35. Ministerio de Agricultura, Ganadería, Acuacultura y Pesca (2010). Otorgamiento de Personería Jurídica a la UNION NACIONAL DE ASOCIACIONES DE PEQUEÑOS PRODUCTORES AGROPECUARIOS CERTIFICADOS EN COMERCIO JUSTO DEL ECUADOR «CECJ» Prov del Oro. Recuperado de http://www.agricultura.gob.ec/acuerdos-ministeriales/

36. Ministerio de Agricultura y Ganadería, Acuacultura y Pesca. (2014). Boletín situacional cacao. Coordinación general del sistema de información nacional. Recuperado de http://sinagap.agricultura.gob.ec/phocadownloadpap/cultivo/2014/db oletin-situacional-de-cacao-2014-actualizado.pdf

37. Morse, J. \& Niehaus, L.(2016).Mixed Methods Design:Principles and Procedures.New York, EE.UU:Routledge Taylor \& Francis Group.

38. Moser,C. \& Kalton, G. (2016). Survey Methods in Social Investigation. New York, EE.UU: Routledge, Taylor \& Francis Group.

39. Oficina regional para Latinoamérica de la Organización Mundial de Comercio Justo

WFTO-LA. (2017). Quienes somos. Recuperado de http://wftola.org/wfto-la/quienes-omos/

40. Onwuegbuzie, A. J., \& Corrigan, J. A. (2014). Improving the Quality of Mixed Research Reports in the Field of Human Resource Development and Beyond: A Call for Rigor as an Ethical Practice. Human Resource Development Quarterly, 25(3), 273-299. doi: $10.1002 / \mathrm{hrdq} .21197$

41. Organización de Naciones Unidas (2015). Objetivos de Desarrollo del Milenio. http://www.un.org/es/millenniumgoals/pdf/2015/mdg-report2015_spanish.pdf

42. Organización de Naciones Unidas (2015). Agenda 2030 para el Desarrollo 
http://www.un.org/sustainabledevelopment/es/2015/09/la-asambleageneral-adopta-la-agenda-2030-para-el-desarrollo-sostenible/

43. Rubin, A. \& Babbie,E. (2017). Research Methods for Social Work. Boston, EE.UU: Cencage Learning.

44. Serrano, J., \& Benzaquén, I. (2017). La frontera de posibilidades de desigualdad en América Latina. El Trimestre Económico, 84(334), 427-461

45. World Fair Trade Organization (2017). History of fair trade. Recuperado de htps://wfto.com/about-us/history-wfto/history-fairtrade

46. Zohrabi, M. (2013).Mixed Method Research: Instruments, Validity, Reliability and Reporting Findings. Theory and practice in Language Studies.3 (2).254-262. doi:10.4304/tpls.3.2.254-262 